\title{
99m Tc-Mebrofenin Hepatobiliary Scintigraphy with SPECT for the Assessment of Hepatic Function and Liver Functional Volume Before Partial Hepatectomy
}

\author{
Wilmar de Graaf ${ }^{1}$, Krijn P. van Lienden², Thomas M. van Gulik ${ }^{1}$, and Roelof J. Bennink ${ }^{3}$ \\ ${ }^{I}$ Department of Surgery, Academic Medical Center, Amsterdam, The Netherlands; ${ }^{2}$ Department of Radiology, Academic Medical \\ Center, Amsterdam, The Netherlands; and ${ }^{3}$ Department of Nuclear Medicine, Academic Medical Center, Amsterdam, \\ The Netherlands
}

Preoperative evaluation of future remnant liver (FRL) function is crucial in the determination of whether a patient can safely undergo liver resection. Although dynamic 99mTc-mebrofenin hepatobiliary scintigraphy (HBS) is used to measure FRL function, 2-dimensional planar images lack the ability to assess segmental liver function. Modern SPECT/CT cameras combine dynamic $99 \mathrm{mTC}$-mebrofenin HBS with additional SPECT and the anatomic information of the CT scan. The aim of this study was to evaluate the additional value of $99 \mathrm{mTc}$-mebrofenin SPECT for the measurement of segmental liver function and liver functional volume. Methods: Preoperative CT volumetry and $99 \mathrm{mTc}-$ mebrofenin HBS with SPECT were performed in 36 patients undergoing liver resection. In 18 patients, postoperative 99mTc-mebrofenin HBS with SPECT was performed within $3 \mathrm{~d}$ after operation. Dual-head dynamic acquisitions were used to calculate FRL function using anterior and geometric mean (Gmean) datasets. Total and FRL functional liver volumes were measured by SPECT. Results: Because of the anatomic position of the liver, the anterior projection resulted in an underestimation of FRL function in patients undergoing left hemihepatectomy. In patients with normal liver parenchyma, total functional liver volume was comparable to total liver volume measured by CT volumetry, indicating that ${ }^{99 \mathrm{~m} T c-m e b r o f e n i n ~ S P E C T}$ is an accurate method to measure hepatic volume. In compromised livers, compared with normal livers, FRL function per cubic centimeter of liver volume was significantly less. In addition, liver function was not distributed homogeneously, with the segments to be resected relatively more affected. FRL function, measured by a combination of SPECT and dynamic HBS, was able to accurately predict actual postoperative remnant liver function. Conclusion: The Gmean dataset is recommended for the assessment of hepatic function by dynamic planar ${ }^{99 m}$ Tc-mebrofenin HBS. The combination of SPECT data with the dynamic uptake function measured by planar HBS provides valuable visible and quantitative information regarding segmental liver function and is an accurate measure for FRL function.

Received Aug. 24, 2009; revision accepted Oct. 26, 2009.

For correspondence or reprints contact: Thomas M. van Gulik, Department of Surgery, Academic Medical Center, Meibergdreef 9, IWO-1 1105 AZ, Amsterdam, The Netherlands.

E-mail: t.m.vangulik@amc.uva.nl

COPYRIGHT @ 2010 by the Society of Nuclear Medicine, Inc.
Key Words: CT volumetry; liver volume; future remnant liver; liver function

J Nucl Med 2010; 51:229-236

DOI: 10.2967/jnumed.109.069724

$\mathbf{T}$ he preoperative evaluation of future remnant liver (FRL) function is crucial for the identification of patients with an increased risk of postoperative liver failure before major liver resection or living donor liver transplantation. Major liver resection can cause insufficient postoperative remnant liver function leading to postoperative liver failure, particularly in patients with parenchymal liver disease (1). The presence of parenchymal liver disease is of growing interest because of the rising number of patients presenting with steatosis or pretreatment with neoadjuvant chemotherapy (2).

FRL volume (FRL-V), measured by CT volumetry, is used as an indirect measurement of liver function (3-5) and is currently the established method to determine whether a patient can safely undergo liver resection. Multiphasic contrast-enhanced CT (CeCT) scans have the advantage of high-resolution diagnostic images that enable accurate measurements of segmental liver volume using portal and hepatic veins as landmarks for segmental division. These types of scans, however, provide no information on the quality of the liver parenchyma in terms of functional capacity and may, therefore, not reflect liver function (6). When using CT volumetry, 2 distinct cutoff values for patients with compromised (due to steatosis, cholestasis, or fibrosis) or noncompromised livers are required for accurate preoperative risk assessment; therefore, CT volumetry is reliable only when the quality of the liver parenchyma is known.

Dynamic ${ }^{99 \mathrm{~m}} \mathrm{Tc}-\mathrm{meb}$ rofenin hepatobiliary scintigraphy (HBS) has the ability to measure total liver function (TL-F) 
and FRL function (FRL-F) (6-8). It incorporates the presence of underlying parenchymal liver disease, as indicated by significantly less liver function in patients with a compromised liver (6). ${ }^{99 \mathrm{~m}} \mathrm{Tc}-$ mebrofenin HBS has the advantage that a single cutoff value for the prediction of liver failure suffices in patients with either normal or compromised liver parenchyma. In patients with unknown quality of liver parenchyma, preoperative dynamic HBS is therefore more valuable than CT volumetry in selecting those with increased risk of postoperative liver failure $(6,8)$.

In previous studies, a single-head $\gamma$-camera was used for dynamic HBS, which permits anterior or posterior projections of the liver only $(7,9)$. Because of the anatomic position of the liver, the left hemiliver is situated more anteriorly, potentially leading to an overestimation of segmental left liver function in the anterior projection. The increasing availability of dual-head rotating $\gamma$-cameras enables simultaneous data acquisition of the anterior and posterior projections, from which a geometric mean (Gmean) dataset can be calculated, thereby reducing the attenuation bias. In addition, fast 3-dimensional SPECT can be performed.

Although dynamic ${ }^{99 \mathrm{~m}}$ Tc-mebrofenin HBS has the possibility to measure regional liver function, the 2-dimensional planar images lack the ability to assess liver function on the segmental level. With a SPECT/CT camera, the functional data from ${ }^{99 \mathrm{~m}} \mathrm{Tc}$-mebrofenin SPECT can be combined with the anatomic information from the CT scan, enabling the measurement of segmental liver function using the CT scan as reference for accurate delineation of liver segments. In addition to segmental liver function, ${ }^{99 \mathrm{~m}} \mathrm{Tc}-$ mebrofenin SPECT could be used to measure hepatic volume. Liver volume calculated with ${ }^{99 \mathrm{~m}} \mathrm{Tc}$-mebrofenin SPECT represents the functional volume because only parts of the liver with functional ${ }^{99 \mathrm{~m}} \mathrm{Tc}$-mebrofenin uptake are included in the volume, and regions with minimal or no uptake are excluded.

The aim of this study was to assess the additional value of ${ }^{99 m}$ Tc-mebrofenin SPECT with low-dose CT for the measurement of segmental liver function and liver functional volume in patients undergoing partial liver resection.

\section{MATERIALS AND METHODS}

\section{Patients}

Between July 2004 and September 2007, 117 patients underwent a partial hepatectomy. Inclusion criteria for this study were liver resection of 2 or more Couinaud segments, preoperative CeCT scan, and ${ }^{99 \mathrm{~m}}$ Tc-mebrofenin HBS combined with SPECT. Patients with preoperative portal vein embolization were excluded. Hence, a group of 36 patients was retrospectively analyzed. Types of resection are summarized in Table 1. Histopathology of the resection specimen was assessed by an experienced hepatopathologist, taking into account features of fibrosis, cholestasis, steatosis, and chronic inflammation. On the basis of the histology, patients were divided into 2 groups: 1 with a normal liver parenchyma and 1 with a compromised liver parenchyma.

\begin{tabular}{lc}
\hline TABLE 1. Patient Characteristics & \\
\multicolumn{1}{c}{ Characteristic } & Value \\
Demographics & \\
Male:female $(n)$ & $17: 19$ \\
Age (y) & \\
Mean \pm SD & $58.5 \pm 12.5$ \\
Range & $33-78$ \\
Body mass index & $25.1 \pm 2.1$ \\
Diagnosis $(n)$ & \\
Cholangiocarcinoma & 14 \\
Liver metastases & 11 \\
Benign biliary strictures & 4 \\
Hepatocellular carcinoma & 3 \\
Cyst adenoma & 2 \\
Adenoma & 1 \\
Caroli syndrome & 1 \\
Compromised liver (yes/no) $(n)$ & $21 / 15$ \\
Fibrosis & 2 \\
Biliary fibrosis & 4 \\
Cholestasis & 2 \\
Steatosis & 2 \\
Chronic inflammation & 2 \\
Combined disease & 9 \\
Resection type ( $n$ ) & \\
Bisegmentectomy & $14(39 \%)$ \\
Trisegmentectomy & $6(\%)$ \\
Left hemihepatectomy & \\
Extended left hemihepatectomy & $11 \%)$ \\
Right hemihepatectomy & $1(28 \%)$ \\
Extended right hemihepatectomy & $1(3 \%)$ \\
& \\
\hline
\end{tabular}

\section{CT Volumetry}

Four-phase CeCT scans were obtained with a multislice helical scanner (Philips). Three-dimensional reconstructions of the liver were made from reconstructed 5-mm-thick axial slices using the portal phase. The total liver, tumor masses, and FRL were manually delineated using portal and hepatic veins as landmarks for segmental division (Fig. 1A). Integrated software was used to calculate total liver volume (TL-V), tumor volume (TV), and FRL-V. The nontumorous total liver volume ( $\left.{ }^{\mathrm{NT}} \mathrm{TL}-\mathrm{V}\right)$ was calculated by excluding the TV from the TL-V. The FRL-V, expressed as a percentage of the ${ }^{\mathrm{NT}} \mathrm{TL}-\mathrm{V}$, was calculated using the formula: $\% \mathrm{FRL}-\mathrm{V}=\frac{\mathrm{FRL}-\mathrm{V}}{\mathrm{NT} \mathrm{TL}-\mathrm{V}} \times 100 \%$.

\section{HBS Imaging}

HBS was performed with ${ }^{99 m}$ Tc-labeled (2,4,6 trimethyl-3bromo)iminodiacetic acid $\left({ }^{99 \mathrm{~m}} \mathrm{Tc}-\right.$ mebrofenin [Bridatec]; GE Healthcare). Patients were positioned supine on the imaging table, with a large-field-of-view (FOV) SPECT/CT camera (Infinia II; GE Healthcare) positioned over the liver and heart region. The SPECT/CT camera was equipped with low-energy high-resolution collimators. First, a dynamic acquisition (36 frames of $10 \mathrm{~s} /$ frame, 128 matrix), which was used for the calculation of the hepatic uptake function, was obtained immediately after the intravenous administration of $200 \mathrm{MBq}$ of ${ }^{99 \mathrm{~m}} \mathrm{Tc}$-mebrofenin. Subsequently, a fast SPECT acquisition was performed (60 projections of $8 \mathrm{~s} /$ projection, 128 matrix), centered on the peak of the hepatic timeactivity curve, which was used for the 3-dimensional assessment of liver function and calculation of functional liver volume. Immediately after SPECT, a low-dose non-contrast-enhanced $\mathrm{CT}\left(\mathrm{CT}_{\text {low }}\right)$ scan was obtained for attenuation correction and 


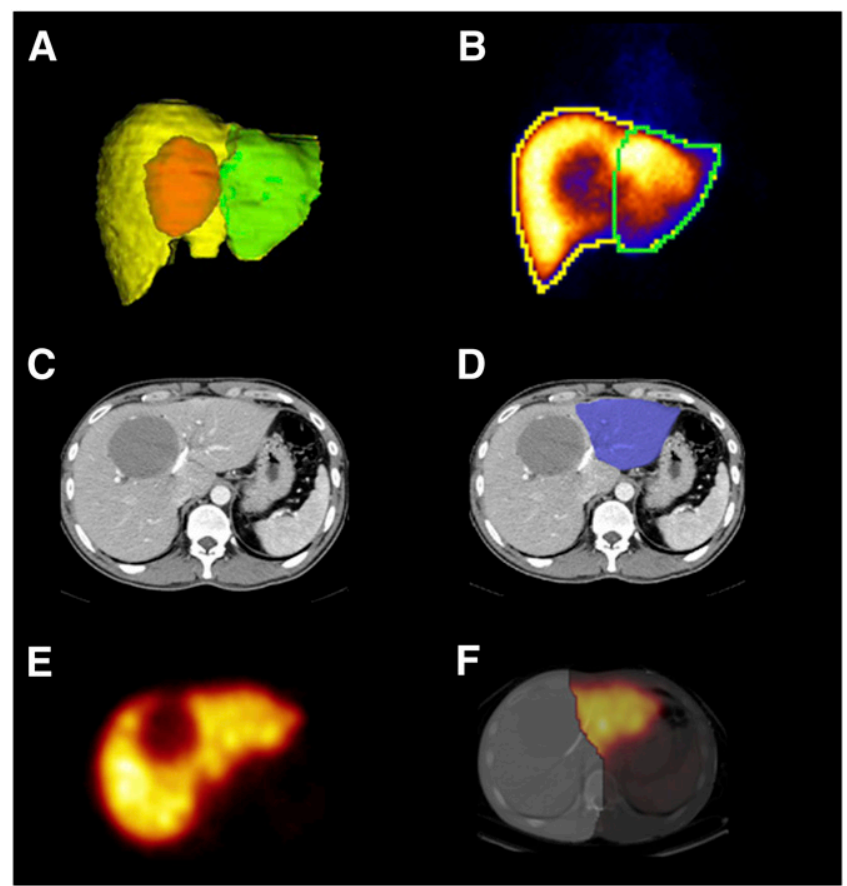

FIGURE 1. Different imaging techniques. Anterior projection of $\mathrm{CeCT}$ reconstruction $(\mathrm{A})$ was used as guideline for delineating FRL on planar dynamic ${ }^{99 m}$ Tc-mebrofenin HBS images (B). Portal and hepatic veins were used as landmarks for delineation of FRL CeCT scans ( $C$ and D). On SPECT image, $\mathrm{FRL}$ was manually outlined on $\mathrm{CT}_{\text {low }}$ scans linked to SPECT images ( $E$ and F). Delineated FRL of CeCT scans was used as constant reference (D).

anatomic mapping on the same gantry, without moving the patient. Finally, a second dynamic acquisition (15 frames of $60 \mathrm{~s} /$ frame, 128 matrix) was obtained to evaluate biliary excretion. Data were processed on a workstation (MultiModality; Hermes Medical Solutions). In 18 patients, postoperative ${ }^{99 \mathrm{~m} T c-m e b r o f e n i n ~ H B S}$ with SPECT/CT $\mathrm{C}_{\text {low }}$ was performed within $3 \mathrm{~d}$ after the operation to measure actual remnant liver function and functional volume.

\section{Calculations of Dynamic HBS Parameters}

The first dual-head dynamic acquisition was used to calculate the hepatic ${ }^{99 \mathrm{~m}} \mathrm{Tc}$-mebrofenin uptake rate using the dataset of the anterior projection and the Gmean dataset. Gmean was calculated using the formula Gmean $=\sqrt{\text { anterior } \times \text { posterior }}$ in which anterior is the data from the anterior projection and posterior from the posterior projection. Regions of interest (ROI) were drawn around the liver, heart, large vessels within the mediastinum (serving as blood pool), and total FOV (indicative of total body activity). ROIs were saved to make sure that identical ROIs were used for the anterior and Gmean datasets. Generation of 3 different timeactivity curves was based on ROIs of the liver, blood pool, and total FOV. With these 3 parameters, the liver uptake rate was calculated as described by Ekman et al. (10) and expressed as percentage per minute $(\% / \mathrm{min})$. The hepatic ${ }^{99 \mathrm{~m}} \mathrm{Tc}-\mathrm{meb} r$ fenin uptake rate was calculated using scanned radioactivity values acquired between 150 and $350 \mathrm{~s}$ after injection, to ensure that calculations were made during a phase of homogeneous distribution of the agent in the blood pool and before the rapid phase of hepatic excretion. To compensate for differences in individual metabolic requirements, total liver ${ }^{99 \mathrm{~m}} \mathrm{Tc}$-mebrofenin uptake rate (\%/min), representing TL-F, was divided by the body surface area and expressed as $\% / \mathrm{min} / \mathrm{m}^{2}$. For comparison of parameters within the same patient, original data $(\% / \mathrm{min})$ were used. TL-F was calculated for both the anterior and the Gmean datasets.

For the FRL (uptake) function (FRL-F), an ROI based on the performed resection was drawn around the FRL by 2 independent investigators. The round ligament was used as the border between segments 3 and 4. Cantlie's line, projected on the liver surface as a plane between the middle of the gallbladder fossa (visible in the late phase of the scintigraphy) and the inferior caval vein, was used as a border between the right and the left liver lobes. In addition, the anterior projection of the CT volumetry was used as a guideline for delineating the FRL (Figs. 1A and 1B). FRL-F was calculated by dividing the summed counts (150-350 s after injection) within the delineated FRL by the total liver counts within the same time frame and multiplying this factor by the total liver ${ }^{99 \mathrm{~m}} \mathrm{Tc}$-mebrofenin uptake rate expressed as $\% / \mathrm{min}$ or $\% / \mathrm{min} /$ $\mathrm{m}^{2}$. FRL-F was calculated for both the anterior (AnteriorFRL-F) and the Gmean (GmeanFRL-F) datasets.

To determine whether the anterior dataset overestimated the function of the left liver segments because of its anatomic position, patients were divided into a group undergoing (extended) right hemihepatectomy (including segments 4-8), with the left hemiliver as FRL $(n=20)$, and a group undergoing a left hemihepatectomy (including segments $2-4)(n=11)$. FRL-F was expressed as the ratio of TL-F for both Gmean (GmeanFRL-F ratio) and anterior data ( ${ }^{\text {Anterior } F R L-F ~ r a t i o) ~ u s i n g ~ i d e n t i c a l ~ R O I s . ~}$ The difference in uptake rate between the anterior and the Gmean datasets was calculated. Positive values indicate an overestimation of the anterior data, and negative values indicate an underestimation, compared with Gmean data.

\section{Calculation of SPECT Parameters}

After the uptake phase, ${ }^{99 \mathrm{~m}} \mathrm{Tc}$-mebrofenin is excreted into the bile. The SPECT acquisition was centered around the peak of the hepatic time-activity curve, because the amount of radioactivity within the liver is relatively stable during this phase. In some patients with a fast hepatic uptake, biliary excretion was already visible during the SPECT phase. Accumulation of radioactivity in the small bile ducts results in voxels with relatively high counts, disturbing threshold-based calculations of total and regional liver function and volume. Therefore, the activity within the extrahepatic bile ducts was manually removed using a masking tool. For the intrahepatic bile ducts, 3 ROIs were drawn around typical bile ducts to determine the minimal and maximal voxel count within the bile ducts. Subsequently, 3 ROIs were placed in surrounding normal liver tissue to measure the average voxel count value of liver tissue. The intrahepatic bile ducts were automatically replaced by the average count density of normal liver tissue using these values. Subsequently, an outline extraction method (with a threshold of $30 \%$ of the maximal voxel count value) was applied to automatically outline the liver and calculate total functional liver volume (TL-FV). The FRL was manually outlined on the $\mathrm{CT}_{\text {low }}$ linked to the SPECT images. The delineated FRL of the CeCT scan was used as a constant reference (Figs. 1D and 1E). FRL functional volume (FRL-FV) was subsequently calculated using the same threshold. FRL-FV, expressed as percentage of TL$\mathrm{FV}$, was calculated using the formula: 


$$
\% \mathrm{FRL}-\mathrm{FV}=\frac{\mathrm{FRL}-\mathrm{FV}}{\mathrm{TF}-\mathrm{FV}} \times 100 \% .
$$

The percentage of counts within the FRL (SPECT\%FRL-C) is calculated by dividing the counts within the FRL by the total counts within the entire liver using the formula:

$$
\% \mathrm{FRL}-\mathrm{C}=\frac{\mathrm{FRL} \text { counts }}{\text { Total liver counts }} \times 100 \%
$$

SPECT\%FRL-C reflects the function of the FRL relative to the TL-F. For the actual calculation of the FRL-F using the SPECT

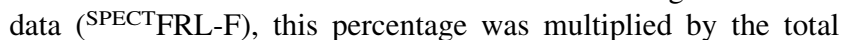
liver ${ }^{99 \mathrm{~m}} \mathrm{Tc}$-mebrofenin uptake rate as measured by the Gmean dataset of the dynamic HBS. To compensate for the size differences between individual FRLs, the SPECTFRL-F was divided by the FRL-V, as measured by the CeCT.

\section{Statistical Analysis}

Statistical analysis was performed with GraphPad Prism, version 4.01 (GraphPad Software) and Statistical Package for Social Sciences (version 14.02; SPSS Inc.). Correlation between variables was tested using the Pearson correlation coefficient. For the comparison between patients with a compromised and patients with a noncompromised liver, the slope coefficient of the linear regression curve was calculated. Continuous data were compared by independent-sample $t$ test or paired $t$ test and expressed as mean \pm SD. All statistical tests were 2-tailed, and differences were considered significant at a $P$ value less than or equal to 0.05 .

\section{RESULTS}

\section{Patient Characteristics}

In this study, 36 patients were included. Patient characteristics are shown in Table 1. Twenty-one patients had a compromised liver based on the histopathology of the resection specimen including severe fibrosis $(n=2)$, biliary fibrosis $(n=4)$, severe cholestasis $(n=2)$, steatosis $(>30 \%$ of the hepatocytes affected) $(n=2)$, chronic inflammation $(n=2)$, or a combination of these diseases $(n=9)$.

\section{Dynamic HBS}

In patients undergoing a right-sided hemihepatectomy (left hemiliver as FRL), the AnteriorFRL-F ratio-compared with the Gmean FRL-F ratio-resulted in an indisputable overestimation of the FRL, whereas an underestimation was seen in patients undergoing a left hemihepatectomy (Fig. 2A). When the FRL-F was calculated, the AnteriorFRL$\mathrm{F}$ was significantly smaller than the GmeanFRL-F in patients undergoing a left hemihepatectomy (Fig. 2B). In patients undergoing a right hemihepatectomy, the relative overestimation as indicated by the AnteriorFRL-F ratio was partially compensated by the significantly smaller total liver function, as calculated by the anterior dataset $(7.71 \% \pm$ $\left.1.19 \% / \mathrm{min} / \mathrm{m}^{2}\right)$ versus the Gmean dataset $(8.76 \% \pm 1.23 \% /$ $\left.\mathrm{min} / \mathrm{m}^{2}\right)(P<0.0001$ paired $t$ test $)$, resulting in only a small, but significant, difference between FRL-Fs of the 2 datasets (mean difference of $0.1 \% / \mathrm{min} / \mathrm{m}^{2}, P<0.0001$ paired $t$ test).

TL-F was significantly less in compromised, compared with noncompromised, livers for both the anterior $(7.4 \% /$ $\mathrm{min} / \mathrm{m}^{2}$ vs. $\left.8.7 \% / \mathrm{min} / \mathrm{m}^{2}\right)$ and the Gmean data $(8.7 \% / \mathrm{min} /$ $\mathrm{m}^{2}$ vs. $9.9 \% / \mathrm{min} / \mathrm{m}^{2}$ ). Patients with a normal liver parenchyma demonstrated a slightly better correlation between FRL-V and FRL-F than did patients with a compromised liver (anterior: Pearson $r=0.81$ vs. Pearson $r=0.73$; Gmean: Pearson $r=0.85$ vs. $r=0.81$ ). Moreover, the slope coefficient of the linear regression curve indicated that FRL-V was associated with a significantly reduced FRL-F in compromised livers versus noncompromised livers $(P=0.008$, ANCOVA test $)$.

In 18 patients, a postoperative HBS was performed within 1-3 d after the operation to measure actual remnant liver function ( ${ }^{\text {Actual }}$ RL-F). Although FRL-F measured by Gmean dataset correlated strongly with Actual RL-F, it slightly underestimated the Actual RL-F with $0.62 \% / \mathrm{min} / \mathrm{m}^{2}$ $(P=0.009)$ (Table 2).

Morphologic Liver Volume and Functional Liver Volume

The TL-FV (measured by SPECT) was compared with morphologic ${ }^{\mathrm{NT}} \mathrm{TL}-\mathrm{V}$ (measured by CeCT volumetry). The TL-FV had a strong and significant correlation with the ${ }^{\mathrm{NT} T L}-\mathrm{V}$ (Pearson $r=0.85$ ). The values of ${ }^{\mathrm{NT} T L}-\mathrm{V}$ and TL-FV were similar in patients with a normal liver parenchyma, indicating the accuracy of ${ }^{99 \mathrm{~m}} \mathrm{Tc}$-mebrofenin SPECT for the measurement of hepatic volume $(1,432.2 \pm$ 315.9 vs. $1,481.2 \pm 301.5$, mean difference; $49 \mathrm{~mL}, P=$ 0.198). However, in patients with a compromised liver parenchyma TL-FV was significantly less than ${ }^{\mathrm{NT}} \mathrm{TL}-\mathrm{V}$

FIGURE 2. FRL-F, calculated by dynamic ${ }^{99 m}$ Tc-mebrofenin HBS using anterior and Gmean datasets. In patients undergoing right-sided hemihepatectomy (with left hemiliver as FRL), AnteriorFRL-F ratio demonstrated clear overestimation of FRL, and underestimation was seen in patients undergoing left hemihepatectomy (A). However, when FRL-F was calculated, anterior projection demonstrated only clear underestimation in patients undergoing left hemihepatectomy $(\mathrm{B})$.

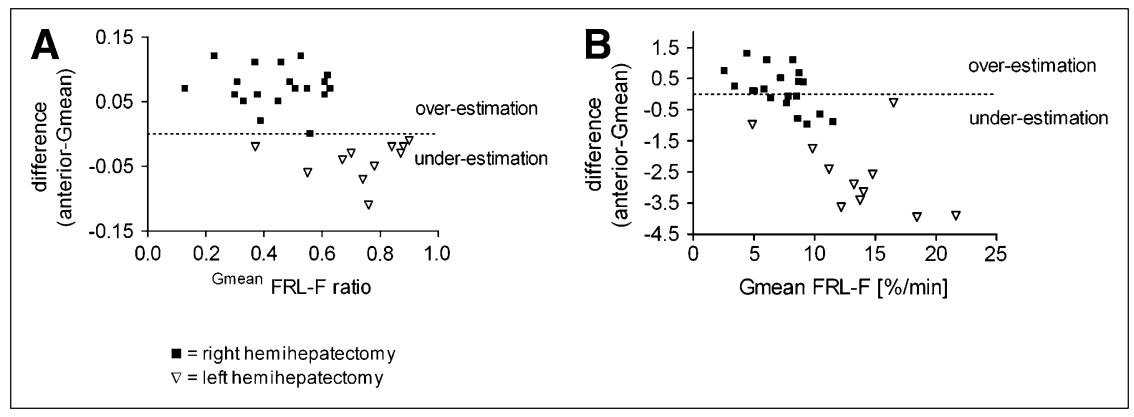


TABLE 2. Preoperative FRL-F Parameter in Relation to Actual Postoperative Remnant Liver Function (ActualRL-F) Measured by Gmean Dataset

\begin{tabular}{|c|c|c|c|c|}
\hline Preoperative parameter & Postoperative parameter & Pearson $r$ & Difference & $P$ \\
\hline Gmean FRL-F & Actual $R L-F^{*}$ & 0.96 & $-0.62 \% / \mathrm{min} / \mathrm{m}^{2}$ & $0.009^{\dagger}$ \\
\hline SPECTFRL-F & Actual $R L-F^{*}$ & 0.94 & $-0.47 \% / \mathrm{min} / \mathrm{m}^{2}$ & 0.068 \\
\hline${ }^{\mathrm{CT}}$ FRL-Function & Actual $\mathrm{RL}-\mathrm{F}^{*}$ & 0.96 & $-2.56 \% / \mathrm{min} / \mathrm{m}^{2}$ & $<0.0001^{\dagger}$ \\
\hline
\end{tabular}

$(1,787.9 \pm 391.5$ vs. $2,000.1 \pm 522.0$, mean difference; $212 \mathrm{~mL}, P=0.006$ ).

In contrast to liver function, ${ }^{\mathrm{NT}} \mathrm{TL}-\mathrm{V}$ was significantly larger in patients with a compromised liver than in patients with a noncompromised liver $\left(1,022 \pm 225 \mathrm{~mL} / \mathrm{m}^{2}\right.$ vs. $853 \pm 163 \mathrm{~mL} / \mathrm{m}^{2}$ ), whereas TL-FV did not significantly differ between these 2 patient groups $\left(824 \pm 168 \mathrm{~mL} / \mathrm{m}^{2}\right.$ vs. $916 \pm 171 \mathrm{~mL} / \mathrm{m}^{2}$ ).

Focusing on the volume of the FRL, a strong correlation was found between FRL-FV and FRL-V (Pearson $r=$ 0.95), with a similar correlation coefficient for both patients with a compromised and patients with a noncompromised liver parenchyma and a similar slope coefficient of the linear regression curve $(P=0.77)$. In addition, the absolute values of FRL-FV were comparable to the values of the FRL-V in both patient groups, indicating that the presence of a compromised liver had not affected the FRL-FV.

The \%FRL-V is a clinically important tool to decide on the resectability of candidates for partial hepatectomy. Analyzing the 2 patient groups separately, \%FRL-FV was comparable to \%FRL-V in patients with a normal liver parenchyma $(56.9 \% \pm 18.9 \%$ vs. $57.2 \% \pm 18.5 \%, P=$ 0.84 , paired $t$ test), whereas \%FRL-FV was significantly larger than \%FRL-V in compromised patients $(58.6 \% \pm$ $22.2 \%$ vs. $55.0 \% \pm 19.4 \%, P=0.006$ ), suggesting that the presence of a compromised liver parenchyma had a greater impact on the functional volume of the segments to be resected than on the FRL.

Postoperative SPECT was performed on 18 patients to measure ActualRL-FV. Although the correlation between FRL-FV and ActualRL-FV was strong (Pearson $r=0.90$ ), the ActualRL-FV was significantly larger than the preoperative prediction (mean difference, $188.5 \mathrm{~mL} ; P=0.0012$, paired $t$ test).

\section{Regional Liver Function by SPECT}

The combination of SPECT and CT images provided valuable visual information on the distribution of function within the liver. The absence of ${ }^{99 \mathrm{~m}} \mathrm{Tc}$-mebrofenin uptake in liver tumors made them clearly visible (Fig. 1E). For the regional distribution of liver function, the FRL counts were expressed as a percentage of total liver counts (SPECT\%FRLC). Although SPECT\%FRL-C correlated strongly with the
\%FRL-V (Pearson $r=0.95$ ), a discrepancy of more than $10 \%$ between the 2 parameters was seen in 9 patients, 7 of whom had a compromised liver. The latter suggests that in these patients, liver function was not distributed homogeneously over the liver volume because of disturbances of regional liver function such as biliary obstruction or tumor compression on the surrounding liver tissue and vessels. The differences in regional liver function of these 9 patients are depicted in Figure 3. In 8 of these patients (89\%), the function within the segments to be resected was more affected than the function within the FRL.

In patients with a compromised liver, ${ }^{\text {SPECT } \% \text { FRL-C was }}$ significantly larger than \%FRL-V $(60.0 \% \pm 24.3 \%$ vs. $55.0 \% \pm 19.4 \%, P=0.011$ ), whereas no difference was found between SPECT\%FRL-C and \%FRL-FV, measured with the outline extraction method.

The SPECT\%FRL-C provides information on the distribution of function within the liver volume, but it does not represent the actual regional function of the liver. Therefore, the SPECTFRL-F was calculated by multiplying the SPECT\%FRL-C by the total liver ${ }^{99 \mathrm{~m}} \mathrm{Tc}$-mebrofenin uptake rate, as measured by the dynamic HBS. This ${ }^{\text {SPECTFRL-F }}$ correlated strongly with ActualRL-F. Moreover, there was no significant difference between ${ }^{2 P E C T}$ FRL-F and Actual RL-F (mean difference, $0.47 \% / \mathrm{min} / \mathrm{m}^{2} ; P=0.068$, paired $t$ test), indicating that ${ }^{\text {SPECT }}$ FRL-F accurately predicted Actual RL-F.

The FRL-F was also calculated using \%FRL-V as measured by $\mathrm{CeCT}$ volumetry multiplied by the total Gmean uptake function from the dynamic HBS (CTFRLF). Although the correlation between CTFRL-F and Actual RL-F was strong (Pearson $r=0.96$ ), the ${ }^{\mathrm{CT}}$ FRL-F significantly underestimated the Actual RL-F, with a mean difference of $2.56 \% / \mathrm{min} / \mathrm{m}^{2}(P<0.0001$, paired $t$ test $)$.

In parallel, SPECT-FVFRL-F was calculated using \%FRLFV multiplied by the Gmean uptake function from the dynamic HBS. This result also underestimated the ActualRL$\mathrm{F}$, although to a lesser extent, with a mean difference of $2.32 \% / \mathrm{min} / \mathrm{m}^{2}(P<0.0001$, paired $t$ test $)$.

Finally, we investigated the function per volume of the FRL, enabling the comparison between the group of patients with parenchymal liver disease and the group with a normal liver parenchyma while compensating for the individual difference in FRL volumes. ${ }^{\text {SPECTFRL-F }} / \mathrm{cm}^{3}$ 


\begin{tabular}{|c|c|c|c|c|c|c|c|}
\hline & Diagnosis & $\begin{array}{l}\text { Impaired } \\
\text { function }\end{array}$ & Explanation & & Mapping CT & $\begin{array}{l}\text { Image } \\
\text { SPECT }\end{array}$ & Diagnostic CT \\
\hline 1 & $\begin{array}{l}\text { Hilar CCC } \\
\text { (klatskin 3) }\end{array}$ & Segm 7,8 & $\begin{array}{l}\text { Cholestasis } \\
\text { with dilated } \\
\text { bile ducts }\end{array}$ & Compromised & & & \\
\hline 2 & $\begin{array}{l}\text { Hilar CCC } \\
\text { (klatskin 3) }\end{array}$ & Segm 2,3 & $\begin{array}{l}\text { Cholestasis } \\
\text { without } \\
\text { dilated bile } \\
\text { ducts }\end{array}$ & Compromised & & & \\
\hline 3 & $\begin{array}{l}\text { Hilar CCC } \\
\text { (klatskin 3) }\end{array}$ & Segm 7 & $\begin{array}{l}\text { Cholestasis } \\
\text { with dilated } \\
\text { bile ducts }\end{array}$ & Compromised & & & \\
\hline 4 & $\begin{array}{l}\text { Intrahepatic } \\
\text { bile stones }\end{array}$ & Segm 2,3 & $\begin{array}{l}\text { Cholestasis } \\
\text { with dilated } \\
\text { bile ducts }\end{array}$ & Compromised & & & \\
\hline 5 & $\begin{array}{l}\text { Metastasis } \\
\text { endometrial } \\
\text { carcinoma }\end{array}$ & $\begin{array}{l}\text { Diffuse left } \\
\text { liver } \\
\text { mainly } \\
\text { segm } 6 \\
\text { distal of } \\
\text { tumor }\end{array}$ & $\begin{array}{l}\text { Tumor } \\
\text { compression } \\
\text { on } \\
\text { surrounding } \\
\text { tissue and } \\
\text { vessels }\end{array}$ & Normal & & & \\
\hline 6 & $\begin{array}{l}\text { Hilar CCC } \\
\text { (klatskin 3) }\end{array}$ & Segm 2,3 & $\begin{array}{l}\text { Cholestasis } \\
\text { with dilated } \\
\text { bile ducts }\end{array}$ & Compromised & & & \\
\hline 7 & $\begin{array}{l}\text { Hilar CCC } \\
\text { (klatskin 3) }\end{array}$ & Segm 2,3 & $\begin{array}{l}\text { Cholestasis } \\
\text { with dilated } \\
\text { bile ducts. } \\
\text { Tumor } \\
\text { invasion left } \\
\text { portal vein }\end{array}$ & Compromised & & & \\
\hline 8 & CRM & $\begin{array}{l}\text { Segm } \\
5,6,7,8\end{array}$ & $\begin{array}{l}\text { Tumor } \\
\text { compression } \\
\text { on } \\
\text { surrounding } \\
\text { tissue and } \\
\text { bile ducts }\end{array}$ & Compromised & & & \\
\hline 9 & CRM & $\begin{array}{l}\text { Segm 2,3 } \\
\text { (FRL) }\end{array}$ & Unknown & Normal & & & \\
\hline
\end{tabular}

liver volume was significantly smaller in patients with a compromised liver.

In patients with a normal liver, the ${ }^{\mathrm{SPECT}} \mathrm{FRL}-\mathrm{F} / \mathrm{cm}^{3}$ liver volume within the segments to be resected and within the FRL was similar, confirming a homogeneous distribution of liver function. Nevertheless, in compromised livers, function per cubic centimeter of volume was significantly less in the segments to be resected than in the FRL, confirming the unequal distribution of liver function and indicating that the resected segments suffer from more functional loss than the FRL.

\section{DISCUSSION}

In this study, we demonstrated the clinical value of

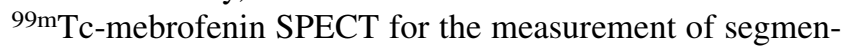
tal liver function and liver functional volume in patients undergoing partial liver resection.
In previous studies, liver function was calculated with dynamic HBS using the anterior projection of the liver (69). This study clearly demonstrated that, because of the anatomic position of the liver and hence different photon attenuation of liver segments, the data from the anterior projection resulted in a relative overestimation of the left hemiliver and an underestimation of the right hemiliver, when data were expressed as a ratio of TL-F. On the other hand, when the function of the FRL was calculated, a clear discrepancy between AnteriorFRL-F and GmeanFRL-F existed in patients undergoing left hemihepatectomy, whereas only a small discrepancy existed in patients undergoing right hemihepatectomy. These discrepancies can be explained by the fact that the right-sided liver segments contribute to approximately two thirds of total liver volume (11), and underestimation of these segments by the anterior projection translates to a significantly smaller TL-F in the 
anterior projection. For the calculation of AnteriorFRL-F in patients undergoing right hemihepatectomy, the relative overestimation of the left hemiliver as indicated by the Anterior FRL-F ratio is consequently compensated by the smaller TL-F. This compensation is relevant because it has been demonstrated that patients undergoing right (extended) hemihepatectomy, especially, are at risk of the development of postoperative liver failure $(12,13)$. Although it is possible to use the anterior projection in these patients, it is recommended that the Gmean data for the dynamic ${ }^{99 \mathrm{~m}} \mathrm{Tc}-\mathrm{mebrofenin}$ HBS be used in the future.

Modern dual-head rotating $\gamma$-cameras enable additional SPECT for the assessment of liver function and liver functional volume within 1 test. The timing of the SPECT is a challenge when a dynamic tracer that is first taken up by the liver and subsequently excreted into the bile is used. We have centered the SPECT around the peak of the hepatic time-activity curve, because the amount of radioactivity within the liver is relatively stable during this phase. In some patients with a fast hepatic uptake, biliary excretion was already visible during the SPECT phase. Accumulation of radioactivity in the small bile ducts results in voxels with relatively high counts, disturbing calculation of total and regional liver function and functional volume. Therefore, we decided to remove the extrahepatic bile ducts by masking on transverse slices. The intrahepatic bile ducts were replaced with the mean voxel count of the liver parenchyma, because true bile duct volume is almost negligible but can be substantial in the SPECT reconstruction because of the relatively low spatial resolution of the SPECT and the relatively high voxel count. This makes ${ }^{99 \mathrm{~m} T c-m e b r o f e n i n ~ S P E C T}$ more complex than ${ }^{99 \mathrm{~m}} \mathrm{Tc}-\mathrm{la}-$ beled galactosyl human serum albumin ( ${ }^{99 \mathrm{~m} T c-G S A}$ ) SPECT, another well-known liver function test showing no early biliary excretion (14-16). ${ }^{99 \mathrm{~m}} \mathrm{Tc}-\mathrm{GSA}$ scintigraphy is a receptor-mediated scintigraphy that is not available for clinical use in Europe and the United States.

The outline extraction method with specific threshold levels is frequently used to calculate functional liver volume from SPECT data $(16,17)$. ${ }^{\mathrm{NT}} \mathrm{TL}-\mathrm{V}$, measured by CeCT volumetry, and TL-FV, measured by ${ }^{99 \mathrm{~m} T c-m e b r o-}$ fenin SPECT, were similar in patients with a normal liver parenchyma, indicating that ${ }^{99 \mathrm{~m} T c-m e b r o f e n i n ~ S P E C T ~ i s ~}$ an accurate method to measure hepatic functional volume. As expected, a compromised liver translated into a significantly smaller TL-FV than ${ }^{\mathrm{NT}} \mathrm{TL}-\mathrm{V}$. Interestingly, the FRL-FV was not significantly different from the FRL-V, indicating that mainly the segments to be resected were affected, leading to loss of functional volume.

A drawback of the outline extraction method is the fact that it does not provide any information regarding the distribution of function within the delineated volume. SPECT images combined with CT images provided valuable visual information on the distribution of function within the liver. For the quantification of the distribution of liver function, we calculated the SPECT\%FRL-C. In patients with a compromised liver, the SPECT\%FRL-C was significantly larger than the \%FRL-V, as determined by $\mathrm{CeCT}$, clearly demonstrating that in patients with a compromised liver, function was not distributed equally among the various liver segments; the segments to be resected were more affected than was the FRL-F. In our patient populations, the proportion of FRL-F is consequently relatively better than suggested by the CeCT scan. Patients in our study were categorized as patients with a normal or a compromised liver on the basis of the histopathology of the resection specimen.

The SPECT count ratio provides information on the distribution of function within the liver, but it does not incorporate the actual regional function of the liver. The actual segmental liver function was, therefore, calculated by multiplying the SPECTFRL count ratio with the total uptake function measured by the Gmean of the dynamic HBS. Preoperative SPECT FRL-F was able to accurately predict the actual postoperative remnant liver function. FRL-F measured by dynamic planar HBS resulted in only a small underestimation of the postoperative remnant liver function. The combination of \%FRL-V acquired from CeCT volumetry with the TL-F from the HBS resulted in a clear underestimation of postoperative remnant liver function. CT volumetry is sometimes combined with TL$\mathrm{F}$ measured by indocyanine green (ICG) clearance test $(18,19)$. Because the ICG clearance test also represents global liver function, this could result in a wrong representation of the FRL-F in a liver with potentially inhomogeneous distribution of function.

To compensate for the size of the FRL, ${ }^{\text {SPECTFRL-F was }}$ divided by FRL-V measured by the CeCT volumetry. Although FRL-V and FRL-FV were similar for patients with either compromised or normal livers, the function within the FRL-V was significantly smaller in compromised livers. In addition, ${ }^{\text {SPECT }}$ FRL-F/ $/ \mathrm{cm}^{3}$ of liver volume was significantly smaller in the resected segments, confirming that liver function was not distributed homogeneously and that the segments to be resected were more affected.

The SPECT/CT camera used in our study allowed only a low-resolution non-contrast-enhanced CT scan, primarily developed as an anatomic reference and for attenuation correction. This low-dose CT is not suitable for use with intravenous contrast and is not a diagnostic tool. We therefore used the separately performed, diagnostic CeCT scan as a reference for the delineation of the FRL. Modern SPECT/CT scans have the ability to combine the SPECT acquisition with a diagnostic multislice $\mathrm{CT}$ scan. This advantage makes it possible to combine a 4-phase CT scan with HBS in which the SPECT data can be used as an additional functional phase, creating a 5-phase SPECT/CT for multimodality preoperative investigation of both liver morphology and liver function. This would enable a safe and accurate prediction of FRL volume and function, forming the basis for clinical decision making in the future. 


\section{CONCLUSION}

When using the dynamic ${ }^{99 \mathrm{~m}} \mathrm{Tc}-\mathrm{mebrofenin} \mathrm{HBS}$, it is recommended that the Gmean dataset be used. Although ${ }^{99 \mathrm{~m} T c-m e b r o f e n i n ~ S P E C T}$ is quite complex because of the rapid biliary excretion of ${ }^{99 \mathrm{~m}} \mathrm{Tc}-$ mebrofenin, it does provide valuable visible and quantitative information on total and segmental liver function. The combination of SPECT data with the dynamic uptake function measured with the planar HBS results in a complete and accurate prediction of the postoperative remnant liver function.

\section{REFERENCES}

1. Shirabe K, Shimada M, Gion T, et al. Postoperative liver failure after major hepatic resection for hepatocellular carcinoma in the modern era with special reference to remnant liver volume. J Am Coll Surg. 1999;188:304-309.

2. Nordlinger B, Sorbye H, Glimelius B, et al. Perioperative chemotherapy with FOLFOX4 and surgery versus surgery alone for resectable liver metastases from colorectal cancer (EORTC Intergroup trial 40983): a randomised controlled trial. Lancet. 2008;371:1007-1016.

3. Shoup M, Gonen M, D'Angelica M, et al. Volumetric analysis predicts hepatic dysfunction in patients undergoing major liver resection. J Gastrointest Surg. 2003; 7:325-330.

4. Vauthey JN, Chaoui A, Do KA, et al. Standardized measurement of the future liver remnant prior to extended liver resection: methodology and clinical associations. Surgery. 2000;127:512-519.

5. Schindl MJ, Redhead DN, Fearon KC, Garden OJ, Wigmore SJ. The value of residual liver volume as a predictor of hepatic dysfunction and infection after major liver resection. Gut. 2005;54:289-296.

6. de Graaf W, Bennink RJ, van Lienden KP, et al. Assessment of future remnant liver function using hepatobiliary scintigraphy in patients undergoing major liver resection. J Gastrointest Surg. November 24, 2009 [Epub ahead of print].

7. Bennink RJ, Dinant S, Erdogan D, et al. Preoperative assessment of postoperative remnant liver function using hepatobiliary scintigraphy. $\mathrm{J}$ Nucl Med. 2004;45:965-971.
8. Dinant S, de Graaf W, Verwer BJ, et al. Risk assessment of posthepatectomy liver failure using hepatobiliary scintigraphy and CT volumetry. J Nucl Med. 2007;48:685-692.

9. Erdogan D, Heijnen BH, Bennink RJ, et al. Preoperative assessment of liver function: a comparison of ${ }^{99 \mathrm{~m}} \mathrm{Tc}$-mebrofenin scintigraphy with indocyanine green clearance test. Liver Int. 2004;24:117-123.

10. Ekman M, Fjalling M, Holmberg S, Person H. IODIDA clearance rate: a method for measuring hepatocyte uptake function. Transplant Proc. 1992;24: 387-388.

11. Abdalla EK, Denys A, Chevalier P, Nemr RA, Vauthey JN. Total and segmental liver volume variations: implications for liver surgery. Surgery. 2004;135:404410.

12. Farges O, Malassagne B, Flejou JF, Balzan S, Sauvanet A, Belghiti J. Risk of major liver resection in patients with underlying chronic liver disease: a reappraisal. Ann Surg. 1999;229:210-215.

13. Jarnagin WR, Gonen M, Fong Y, et al. Improvement in perioperative outcome after hepatic resection: analysis of 1,803 consecutive cases over the past decade. Ann Surg. 2002;236:397-406.

14. de Graaf W, Vetelainen RL de BK, van Vliet AK, van Gulik TM, Bennink RJ. ${ }^{99 m}$ Tc-GSA scintigraphy with SPECT for assessment of hepatic function and functional volume during liver regeneration in a rat model of partial hepatectomy. J Nucl Med. 2008;49:122-128.

15. Kokudo N, Vera DR, Makuuchi M. Clinical application of TcGSA. Nucl Med Biol. 2003;30:845-849.

16. Kwon AH, Matsui Y, Ha-Kawa SK, Kamiyama Y. Functional hepatic volume measured by technetium-99m-galactosyl-human serum albumin liver scintigraphy: comparison between hepatocyte volume and liver volume by computed tomography. Am J Gastroenterol. 2001;96:541-546.

17. Jansen PL, Chamuleau RA, van Leeuwen DJ, Schipper HG, Busemann-Sokole E, van der Heyde MN. Liver regeneration and restoration of liver function after partial hepatectomy in patients with liver tumors. Scand $J$ Gastroenterol. 1990;25:112-118.

18. Mitsumori A, Nagaya I, Kimoto S, et al. Preoperative evaluation of hepatic functional reserve following hepatectomy by technetium- $99 \mathrm{~m}$ galactosyl human serum albumin liver scintigraphy and computed tomography. Eur J Nucl Med. 1998;25:1377-1382.

19. Okochi O, Kaneko T, Sugimoto H, Inoue S, Takeda S, Nakao A. ICG pulse spectrophotometry for perioperative liver function in hepatectomy. J Surg Res. 2002;103:109-113. 